\title{
COMMENT
}

\section{A novel coronavirus (2019-nCoV) causing pneumonia-associated respiratory syndrome}

\author{
Shibo Jiang ${ }^{1}$, Shuai Xia ${ }^{1}$, Tianlei Ying $\mathbb{D}^{1}$ and $\mathrm{Lu} \mathrm{Lu}^{1}$ \\ Cellular \& Molecular Immunology (2020) 17:554; https://doi.org/10.1038/s41423-020-0372-4
}

By the end of 2019, the World Health Organization (WHO) was informed about an outbreak of pneumonia of unknown etiology in Wuhan, China. Some patients were linked to a seafood market, suggesting animal-to-human transmission, but, soon, human-tohuman transmission was confirmed. The pathogen was promptly identified as a novel coronavirus belonging to lineage $B$ betacoronaviruses that also include severe acute respiratory syndrome coronavirus (SARS-CoV), which caused a pandemic in China in 2002/2003, and bat SARS-like coronaviruses.

Since 2012, the outbreak of Middle East respiratory syndrome coronavirus infection starting from Saudi Arabia has resulted in 2494 confirmed cases and 858 deaths in 27 countries, posing ongoing threat to global public health (https://www.who.int/ emergencies/mers-cov/en).

As of 1 February 2020, there were 12,024 confirmed cases with Wuhan pneumonia and 259 deaths, of which 11,860 cases were found in Mainland China and 164 in 26 other countries and territories. The estimated case fatality rate is about $2 \%$, which is much lower than that of SARS (9.6\%), while its transmission rate (R0) is $2-3 \%$ (https://www.worldometers.info/coronavirus), similar to that of SARS (3\%). Like SARS-CoV-infected individuals, patients infected with this novel betacoronavirus showed abnormal findings on chest computed tomography, along with common symptoms that include fever, cough, and myalgia, or fatigue, at the onset of illness. Still, all indications suggest that the acute respiratory syndrome of SARS is, so far, more severe than that manifested by the present pneumonia outbreak. ${ }^{2}$

The novel coronavirus was denoted as "2019-nCoV" by WHO (https://www.who.int/emergencies/diseases/novel-coronavirus2019) and the Wuhan pneumonia was named as "novel coronavirus-infected pneumonia (NCIP)" by Chinese scientists. ${ }^{3}$ However, it will seem illogical if coronavirus is listed after NCIP, i.e., "novel coronavirus-infected pneumonia coronavirus (NCIP-CoV)". Therefore, we suggest renaming NCIP as "pneumonia-associated respiratory syndrome (PARS)" and 2019-nCoV as "PARS coronavirus (PARS-CoV)", similar to SARS-CoV, to retain equivalent terminology.

\section{ADDITIONAL INFORMATION}

Competing interests: The authors declare no competing interests.

\section{REFERENCES}

1. Jiang, S., Du, L. \& Shi Z. An emerging coronavirus causing pneumonia outbreak in Wuhan, China: calling for developing therapeutic and prophylactic strategies. Emerg. Microbes Infect. 9, 275-277 (2020).

2. Huang, C. et al. Clinical features of patients infected with 2019 novel coronavirus in Wuhan, China. Lancet. https://doi.org/10.1016/S0140-6736(20)30183-5 (2020).

3. Li, Q. et al. Early transmission dynamics in Wuhan, China, of novel coronavirusinfected pneumonia. N. Engl. J. Med. https://doi.org/10.1056/NEJMoa2001316 (2020). 